\title{
Nasal hygiene in patients with end-stage renal disease
}

\author{
Zhao Fan $^{1}$ and Baiya $\mathrm{Li}^{2 *}$ \\ ${ }^{1}$ Dialysis Department of Nephrology Hospital, the First Affiliated Hospital of Xi'an Jiaotong University, Xi'an, Shaanxi, 710061, P.R. China \\ ${ }^{2}$ Department of Otorhinolaryngology, the First Affiliated Hospital of Xi'an Jiaotong University, Xi'an, Shaanxi 710061, P.R. China
}

\begin{abstract}
Staphylococcus aureus is the most common endogenous infection in patients with end-stage renal disease (ESRD), and the anterior nares are the most common endogenous $S$. aureus carrier sites. Eliminating nasal $S$. aureus will greatly benefit ESRD patients. However, prophylactic topical nasal usage of mupirocin or any kind of antibiotic is against the principles of antibiotic usage. Nasal irrigation has been demonstrated to significantly increase ciliary clearance and decrease mucous inflammation, and has been proven to be an inexpensive, effective, simple, and safe treatment method in nasal health care. We propose that nasal irrigation might be the appropriate nasal hygiene intervention for ESRD patients and should be routinely applied in ESRD patients, especially those with risk factors.
\end{abstract}

\section{Introduction}

Infection is one of the most common causes of hospitalization, morbidity, and mortality among patients with End-Stage Renal Disease (ESRD) who are undergoing hemodialysis (HD), Peritoneal Dialysis (PD), Continuous Ambulatory Peritoneal Dialysis (CAPD), or kidney transplantations [1-3]. Endogenous gram-positive cocci, especially Staphylococcus aureus (S. aureus), are the most frequently associated microorganism in long-term dialysis patients, and anterior nares are the most common endogenous SA carrier sites [4].

\section{Nasal $S$. aureus is the most common endogenous infective resource of ESRD patients}

Based on sensitivity to methicillin, S. aureus is usually divided into two subclasses, namely methicillin-resistant $S$. aureus (MRSA) and methicillin-sensitive S. aureus (MSSA). 15.1\%-73.9\% ESRD patients carried nasal MSSA while 1.1\%-27.4\% ESRD patients carried MRSA in their noses (Table 1).

Nasal S. aureus will spread to the skin and catheter exits via touching, or to the bronchus and lung via airflow. Hence, the nose is the main original source of endogenous $S$. aureus. Besides, most $S$. aureus nasal carriers are asymptomatic but have greater potential risk of bacteremia than non-carriers. In particular, dialysis patients who are S. aureus nasal carriers usually have poor clinical outcomes, especially elderly patients $[1,2]$. Thus, eliminating nasal $S$. aureus will benefit ESRD patients and reduce the economic burden of both the patients and the government $[1,2]$.

\section{Prophylactic antibiotic is no longer the first choice to eliminate nasal $S$. aureus}

Topical mupirocin application has been proven to be effective in eradicating S. aureus in the nose and catheter exits [5], and has been applied for years [6]. Topical application of mupirocin near catheter exits is rational and necessary because catheterization is an invasive treatment. However, there is no adequate reason for prophylactic nasal application of mupirocin because most $S$. aureus carriers are asymptomatic. Prophylactic antibiotic usage will induce antibiotic resistance and break the balance of nasal flora between $S$. aureus and other microorganisms such as Staphylococcus epidermidis. Therefore, prophylactic topical usage of mupirocin or any kind of antibiotic in ESRD patients is against the principles of antibiotic usage. Meanwhile, screening for nasal $S$. aureus is a time-consuming and economically inefficient process.

\section{Nasal irrigation in patients with end-stage renal disease}

Nasal hygiene of ESRD patients is essential but long-ignored. From otolaryngologists' perspective, we propose that topical nasal irrigation might be the appropriate nasal hygiene intervention for ESRD patients.

Nasal irrigation, also called nasal wash, rinse, douche, and lavage, is a series of adjunctive treatments for patients with chronic sinusitis and a postoperative treatment of other nasal diseases. Abundant data provide evidence that nasal irrigation is an inexpensive, effective, simple, and safe treatment [7-10]. Treatment guidelines in many countries, including China, Europe, and North America, now advocate the use of nasal irrigation for all causes of rhinosinusitis and for postoperative cleaning of the nasal cavities [11].

Nasal irrigation is performed by injecting saline in one nostril and allowing it to drain out of the other nostril, bathing the nasal cavity. In the past century, many trials have been conducted about the irrigating solution components and devices [10]. Currently, a consensus seemed to have been reached that the combination of high-volume and lowpressure devices with hypertonic solutions show optimal outcomes $[11,12]$. Nasal irrigation has no longer been considered as merely an adjunctive treatment and is now becoming increasingly popular in nasal healthcare [12].

The beneficial mechanisms of nasal irrigation is to increase

Correspondence to: Baiya Li, No. 277 Yanta West Road, Xi'an, Shaanxi Province, PR-China, 710061, Tel: 86-177-9182-4589; E-mail: lby0929@163.com

Key words: ESRD patients, dialysis, nasal S.aureus, nasal irrigation

Received: October 28, 2015; Accepted: November 17, 2015; Published: November 20, 2015 
Table 1. Nasal carriage of $S$. aureus and MRSA in ESRD patients.

\begin{tabular}{|c|c|c|c|c|c|c|c|c|}
\hline \multirow[t]{2}{*}{ Sample Size } & \multicolumn{2}{|c|}{$\begin{array}{c}\text { Total } \\
\text { S. aureus }\end{array}$} & \multicolumn{2}{|c|}{ MRSA } & \multirow[t]{2}{*}{ Dialysis types } & \multirow[t]{2}{*}{ Experiment Year } & \multirow[t]{2}{*}{ Country } & \multirow[t]{2}{*}{ Reference } \\
\hline & $\mathbf{n}$ & $\%$ & $\mathbf{n}$ & $\%$ & & & & \\
\hline 87 & 20 & 23.0 & - & - & CAPD & 1984 & UK & {$[18]$} \\
\hline 140 & 63 & 45.0 & - & - & CAPD & 1987 & Belgium & {$[19]$} \\
\hline 146 & 41 & 28.1 & - & - & CAPD & 1989 & Singapore & {$[20]$} \\
\hline 167 & - & - & 28 & 16.8 & CAPD & 1989 & Singapore. & {$[21]$} \\
\hline 129 & 66 & 51.2 & - & - & CAPD & 1992 & Denmark & {$[22]$} \\
\hline 129 & 60 & 46.5 & - & - & CAPD & 1992 & Denmark & {$[22]$} \\
\hline 168 & 100 & 59.5 & - & - & HD & 1992 & Denmark & {$[22]$} \\
\hline 168 & 96 & 57.1 & - & - & HD & 1992 & Denmark & {$[22]$} \\
\hline 172 & 67 & 39.0 & - & - & HD & 1992 & Netherland & {$[6]$} \\
\hline 54 & 31 & 57.4 & - & - & CAPD & 1993 & Netherland & {$[23]$} \\
\hline 138 & 69 & 50.0 & - & - & PD & 1993 & USA & [24] \\
\hline 32 & 12 & 37.5 & - & - & CAPD & 1995 & Denmark & {$[25]$} \\
\hline 24 & 9 & 37.5 & - & - & CAPD & 1996 & USA & {$[26]$} \\
\hline 205 & 78 & 38.0 & 22 & 10.7 & HD & 1997 & Saudi Arabia & {$[15]$} \\
\hline 28 & 16 & 57.1 & - & - & HD & 1998 & Poland & [27] \\
\hline 52 & 30 & 57.7 & - & - & PD & 1998 & Netherland & {$[28]$} \\
\hline 144 & 50 & 34.7 & - & - & HD,CAPD & 1999 & UK & {$[29]$} \\
\hline 71 & 39 & 54.9 & - & - & HD & 2000 & Spain & {$[30]$} \\
\hline 83 & - & - & 2 & 2.4 & $\mathrm{PD}$ & 2002 & TAIWAN & {$[31]$} \\
\hline 198 & - & - & 11 & 5.6 & HD & 2002 & USA & {$[32]$} \\
\hline 509 & - & - & 12 & 2.4 & HD & 2002 & TAIWAN & {$[31]$} \\
\hline 69 & 28 & 40.6 & - & - & HD & 2003 & Iran & {$[33]$} \\
\hline 43 & 12 & 27.9 & - & - & HD & 2004 & Poland & [34] \\
\hline 43 & 12 & 27.9 & 1 & 2.3 & HD & 2004 & Poland & {$[35]$} \\
\hline 136 & 72 & 52.9 & 16 & 11.8 & HD & 2004 & GERMAN & {$[4]$} \\
\hline 261 & 148 & 56.7 & - & - & HD & 2004 & Turkey & [36] \\
\hline 289 & - & - & 34 & 11.8 & HD & 2004 & Germany & {$[5]$} \\
\hline 84 & 31 & 36.9 & 23 & 27.4 & HD & 2006 & Iran & [37] \\
\hline 157 & 26 & 16.6 & 10 & 6.4 & HD & 2006 & USA & [38] \\
\hline 103 & - & - & 12 & 11.7 & HD & 2007 & USA & [39] \\
\hline 120 & 40 & 33.3 & 26 & 21.7 & DIALYSIS & 2007 & USA & [17] \\
\hline 130 & 32 & 24.6 & - & - & HD & 2007 & Iran & {$[40]$} \\
\hline 306 & - & - & 29 & 9.5 & HD & 2007 & TAIWAN & {$[1]$} \\
\hline 541 & 121 & 22.4 & 32 & 5.9 & HD & 2007 & TAIWAN & [16] \\
\hline 54 & 24 & 44.4 & 3 & 5.6 & HD,CAPD & 2008 & Maroc & [41] \\
\hline 54 & 24 & 44.4 & 3 & 5.6 & HD & 2008 & Morocco & [41] \\
\hline 46 & 34 & 73.9 & - & - & TRANSP. & 2009 & Brazil & {$[42]$} \\
\hline 48 & 36 & 75.0 & - & - & TRANSP. & 2009 & Brazil & [42] \\
\hline 70 & 37 & 52.9 & - & - & DIALY. & 2009 & Brazil & [43] \\
\hline 111 & 55 & 49.0 & - & - & DIALY. & 2009 & Brazil & [43] \\
\hline 112 & - & - & 10 & 8.9 & HD & 2009 & JAPAN & [44] \\
\hline 264 & 48 & 18.2 & 14 & 5.3 & DIALYSIS & 2009 & TAIWAN & {$[2]$} \\
\hline 70 & 30 & 42.9 & 1 & 1.4 & HD & 2010 & Morocco. & {$[45]$} \\
\hline 103 & - & - & 4 & 3.9 & HD & 2010 & JAPAN & [44] \\
\hline 184 & 52 & 28.3 & - & - & HD & 2011 & Turkey & [46] \\
\hline 296 & 48 & 16.2 & 20 & 6.8 & HD & 2011 & TAIWAN & [47] \\
\hline 185 & 28 & 15.1 & 2 & 1.1 & HD & 2012 & Turkey & [48] \\
\hline 28 & 16 & 57.1 & - & - & HD & - & Poland & [27] \\
\hline 91 & 34 & 37.4 & - & - & HD & - & Netherland & {$[23]$} \\
\hline 114 & 34 & 29.8 & - & - & HD & - & Marseille & {$[50]$} \\
\hline
\end{tabular}

mucociliary clearance and decrease mucous inflammations, which might include the following aspects: 1) activation of the cilia motility and decreasing the bacterial adhesion, and therefore reducing nasal bacterial attachment; 2) physically flushing away inflammatory mediators, the crust, and other nasal discharges that act as the culture media of bacteria, hence inhibiting bacterial growth [7]. 
Only low-level evidence support the efficacy of nasal irrigation with antibiotics, suggesting that irrigation itself plays a more important role than the additive antibiotics. Nasal irrigation has been proven to decrease antibiotic usage and thereafter reduce antibiotic resistance [9]. Unfortunately, direct data are lacking that show the elimination effect of nasal irrigation on S. aureus.

Given that it is an inexpensive and convenient procedure, nasal irrigation is recommended to all dialysis patients and health-care staff. Moreover, it should be routinely used among patients with risk factors, which at least include the following: 1) antibiotic usage within 3 months before admission[13]; 2) hospitalization during the past 12 months [13]; 3) diagnosis of skin or soft tissue infection at admission [13,14]; 4) human immunodeficiency virus infection [13]; 5) elderly patient ( $\geq 75$ years) $[1,15] ; 6)$ prolonged hospitalization $[1,2]$; 7) congestive heart failure [1,2]; and 8) nursing home admission and nasogastric tube feeding in the last 3 months $[16,17]$.

\section{Conflict of interest}

No conflict of interest relevant to this paper is declared.

\section{Disclosure of grants or other funding}

\section{None}

\section{References}

1. Lai CF, Liao CH, Pai MF, Chu FY, Hsu SP, et al. (2011) Nasal carriage of methicillinresistant Staphylococcus aureus is associated with higher all-cause mortality in hemodialysis patients. Clin J Am Soc Nephrol 6: 167-174. [Crossref]

2. Wang CY, Wu VC, Chen YM, Su CT, Wu KD, et al. (2010) Risk factors for methicillinresistant Staphylococcus aureus colonization among elderly patients with end-stage renal disease in Taiwan. Am J Infect Control 386: 499-500. [Crossref]

3. Hoen B, Paul-Dauphin A, Hestin D, Kessler M (1998) EPIBACDIAL: a multicenter prospective study of risk factors for bacteremia in chronic hemodialysis patients. $J \mathrm{Am}$ Soc Nephrol 9: 869-876. [Crossref]

4. Lederer SR, Riedelsdorf G, Schiffl H (2007) Nasal carriage of meticillin resistant Staphylococcus aureus: the prevalence, patients at risk and the effect of elimination on outcomes among outclinic haemodialysis patients. Eur J Med Res 127: 284-288. [Crossref]

5. Schmid H, Romanos A, Schiffl H, Lederer SR (2013) Persistent nasal methicillinresistant Staphylococcus aureus carriage in hemodialysis outpatients: a predictor of worse outcome. BMC Nephrol 14: 93.

6. Kluytmans JA, Manders MJ, van Bommel E, Verbrugh H (1996) Elimination of nasal carriage of Staphylococcus aureus in hemodialysis patients. Infect Control Hosp Epidemiol 17: 793-797. [Crossref]

7. Papsin B, McTavish A (2003) Saline nasal irrigation: Its role as an adjunct treatment. Can Fam Physician 49: 168-173. [Crossref]

8. Rabago D, Zgierska A (2009) Saline nasal irrigation for upper respiratory conditions. Am Fam Physician 80: 1117-1119. [Crossref]

9. Adappa ND, Wei CC, Palmer JN (2012) Nasal irrigation with or without drugs: the evidence. Curr Opin Otolaryngol Head Neck Surg 20: 53-57. [Crossref]

10. Rudmik L, Soler ZM, Orlandi RR, Stewart MG, Bhattacharyya N, et al. (2011) Early postoperative care following endoscopic sinus surgery: an evidence-based review with recommendations. Int Forum Allergy Rhinol 1: 417-430. [Crossref]

11. Dunn JD, Dion GR, McMains KC (2013) Efficacy of nasal irrigations and nebulizations for nasal symptom relief. Curr Opin Otolaryngol Head Neck Surg 21: 248-251. [Crossref]

12. Brown CL, Graham SM (2004) Nasal irrigations: good or bad? Curr Opin Otolaryngol Head Neck Surg 12: 9-13. [Crossref]

13. Hidron AI, Kourbatova EV, Halvosa JS, Terrell BJ, McDougal LK, et al. (2005) Risk factors for colonization with methicillin-resistant Staphylococcus aureus MRSA) in patients admitted to an urban hospital: emergence of community-associated MRSA nasal carriage. Clin Infect Dis 412: 159-66. [Crossref]
14. Hoen B, Kessler M, Hestin D, Mayeux D (1995) Risk factors for bacterial infections in chronic haemodialysis adult patients: a multicentre prospective survey. Nephrol Dial Transplant 10: 377-381. [Crossref]

15. Saxena AK, Panhotra BR, Chopra R (2004) Advancing age and the risk of nasal carriage of Staphylococcus aureus among patients on long-term hospital-based hemodialysis. Ann Saudi Med 24: 337-342. [Crossref]

16. Wang CY, Wu VC, Wang WJ, Chen YM, Su CT, et al. (2009) Nasal carriage of methicillin-resistant Staphylococcus aureus among patients with end-stage renal disease. Infect Control Hosp Epidemiol 301: 93-4. [Crossref]

17. Wang CY, Wu VC, Wang WJ, Lin YF, Lin YH, et al. (2012) Risk factors for nasal carriage of methicillin-resistant Staphylococcus aureus among patients with end-stage renal disease in Taiwan. J Formos Med Assoc 111: 14-18. [Crossref]

18. Davies SJ, Ogg CS, Cameron JS, Poston S, Noble WC (1989) Staphylococcus aureus nasal carriage, exit-site infection and catheter loss in patients treated with continuous ambulatory peritoneal dialysis CAPD). Perit Dial Int 9: 61-64. [Crossref]

19. Luzar MA, Coles GA, Faller B, Slingeneyer A, Dah GD, et al. (1990) Staphylococcus aureus nasal carriage and infection in patients on continuous ambulatory peritoneal dialysis. N Engl J Med 322: 505-509. [Crossref]

20. Lye WC, Leong SO, van der Straaten J, Lee EJ (1994) Staphylococcus aureus CAPDrelated infections are associated with nasal carriage. Adv Perit Dial 10: 163-165. [Crossref]

21. Lye WC, Leong SO, Lee EJ (1993) Methicillin-resistant Staphylococcus aureus nasal carriage and infections in CAPD. Kidney Int 43: 1357-1362. [Crossref]

22. Zimakoff J, Bangsgaard Pedersen F, Bergen L, Baagø-Nielsen J, Daldorph B, et al (1996) Staphylococcus aureus carriage and infections among patients in four haemoand peritoneal-dialysis centres in Denmark. The Danish Study Group of Peritonitis in Dialysis DASPID). J Hosp Infect 334: 289-300. [Crossref]

23. Wanten GJ, van Oost P, Schneeberger PM, Koolen MI (1996) Nasal carriage and peritonitis by Staphylococcus aureus in patients on continuous ambulatory peritoneal dialysis: a prospective study. Perit Dial Int 16: 352-356. [Crossref]

24. Piraino B, Perlmutter JA, Holley JL, Bernardini J (1993) Staphylococcus aureus peritonitis is associated with Staphylococcus aureus nasal carriage in peritoneal dialysis patients. Perit Dial Int 13: S332-334. [Crossref]

25. Bistrup C, Jensen KT, Kabel B, Pedersen RS (1997) Staphylococcus aureus carriage in adult peritoneal dialysis patients and their spouses. Perit Dial Int 17: 480-485. [Crossref]

26. Mylotte JM, Kahler L, Jackson E (2011) "Pulse" nasal mupirocin maintenance regimen in patients undergoing continuous ambulatory peritoneal dialysis. Infect Control Hosp Epidemiol: 741-745. [Crossref]

27. Koziol-Montewka M, Chudnicka A, Ksiazek A, Majdan M (2001) Rate of Staphylococcus aureus nasal carriage in immunocompromised patients receiving haemodialysis treatment. Int J Antimicrob Agents 182: 193-6. [Crossref]

28. Nouwen JL, Fieren MW, Snijders S, Verbrugh HA, van Belkum A 2005) Persistent not intermittent) nasal carriage of Staphylococcus aureus is the determinant of CPD-related infections. Kidney Int 67: 1084-1092. [Crossref]

29. Peacock SJ, de Silva GD, Justice A, Cowland A, Moore CE, et al. (2002) Comparison of multilocus sequence typing and pulsed-field gel electrophoresis as tools for typing Staphylococcus aureus isolates in a microepidemiological setting. J Clin Microbiol 4010: 3764-70. [Crossref]

30. Peña C, Fernández-Sabe N, Domínguez MA, Pujol M, Martinez-Castelao A, et al (2004) Staphylococcus aureus nasal carriage in patients on haemodialysis: role of cutaneous colonization. J Hosp Infect 58: 20-27. [Crossref]

31. Lu PL, Tsai JC, Chiu YW, Chang FY, Chen YW, et al. (2008) Methicillin-resistan Staphylococcus aureus carriage, infection and transmission in dialysis patients, healthcare workers and their family members. Nephrol Dial Transplant 235: 16591665. [Crossref]

32. Hadley AC, Karchmer TB, Russell GB, McBride DG, Freedman BI (2007) The prevalence of resistant bacterial colonization in chronic hemodialysis patients. Am J Nephrol 27: 352-359. [Crossref]

33. Ghazvini K, Hekmat R (2007) Nasal and skin colonization of Staphylococcus aureus in hemodialysis patients in Northeast of Iran. Iran J Kidney Dis 1: 21-24. [Crossref]

34. Koziol-Montewka M, Szczepanik A, Baranowicz I, Jozwiak L, Ksiazek A. et al. (2006) The investigation of Staphylococcus aureus and coagulase-negative staphylococc 
nasal carriage among patients undergoing haemodialysis. Microbiol Res 1614: 281-7. [Crossref]

35. Bogut A, Kozioł-Montewka M, Baranowicz I, Jóźwiak L, Ksiazek A, et al. (2007) Characterisation of Staphylococcus aureus nasal and skin carriage among patients undergoing haemodialysis treatment. New Microbiol 302: 149-54. [Crossref]

36. Duran N, Ocak S, Eskiocak AF (2006) Staphylococcus aureus nasal carriage among the diabetic and non-diabetic haemodialysis patients. Int J Clin Pract 60: 1204-1209. [Crossref]

37. Ghasemian R, Najafi N, Makhlough A, Khademloo M (2010) Frequency of nasal carriage of Staphylococcus aureus and its antimicrobial resistance pattern in patients on hemodialysis. Iran J Kidney Dis 4: 218-222. [Crossref]

38. Alexander EL, Morgan DJ, Kesh S, Weisenberg SA, Zaleskas JM, et al. (2011) Prevalence, persistence, and microbiology of Staphylococcus aureus nasal carriage among hemodialysis outpatients at a major New York Hospital. Diagn Microbiol Infect Dis 701: 37-44. [Crossref]

39. Patel G, Jenkins SG, Mediavilla JR (2011) Clinical and molecular epidemiology of methicillin-resistant Staphylococcus aureus among patients in an ambulatory hemodialysis center. Infect Control Hosp Epidemiol 329: 881-8. [Crossref]

40. Motamedifar M, Hassanzadeh P, Ghafari N (2010) Relative frequency of Staphylococcal carriage and antibiotic sensitivity of isolated Staphylococci in hemodialysis patients in Shiraz, Iran. Med Princ Pract 195: 379-383. [Crossref]

41. Souly K, Ait el kadi M, Lahmadi K, Biougnach H, Boughaidi A, et al. (2011) Epidemiology and prevention of Staphylococcus aureus nasal carriage in hemodialyzed patients. Med Mal Infect 41: 469-474. [Crossref]

42. Giarola LB, Dos Santos RR, Bedendo J, da Silva Junior WV and Borelli SD (2012) HLA molecules and nasal carriage of Staphylococcus aureus isolated from dialysis and kidney transplant patients at a hospital in Southern Brazil. BMC Res Notes 5: 90. [Crossref]
43. Giarola LB, Dos Santos RR, Tognim MC, Borelli SD, Bedendo J (2012) Carriage frequency, phenotypic and genotypic characteristics of Staphylococcus aureus isolated from dialysis and kidney tranplant patients at a hosptial in northern parana. Braz J Microbiol 433: 923-30.

44. Uehara Y, Kuwahara-Arai K, Hori S, Kikuchi K, Yanai M, et al. (2013) Investigation of nasal meticillin-resistant Staphylococcus aureus carriage in a haemodialysis clinic in Japan. J Hosp Infect 84: 81-84. [Crossref]

45. Oumokhtar B, Elazhari M, Timinouni M, Bendahhou K, Bennani B, et al. (2013) Staphylococcus aureus nasal carriage in a Moroccan dialysis center and isolates characterization. Hemodial Int 17: 542-547. [Crossref]

46. Celik G, Gulcan A, Dikici N, Gulcan E (2011) Prevalence of nasal Staphylococcus aureus carriage in the patients undergoing hemodialysis and evaluation of risk factors and laboratory parameters. Ren Fail 335: 494-498. [Crossref]

47. Kang YC, Tai WC, Yu CC, Kang JH, Huang YC (2012) Methicillin-resistan Staphylococcus aureus nasal carriage among patients receiving hemodialysis in Taiwan: prevalence rate, molecular characterization and de-colonization. BMC Infect Dis 12: 284.

48. Aydogan U, Akbulut H, Gok DE, Yilmaz MI, Yuksel S, et al. (2012) To study the correlation between carrier status of nasal Staphylococcus aureus in patients on haemodialysis with hepatitis C, hepatitis B and their sociodemographic features. West Indian Med J 612: 139-44. [Crossref]

49. Wanten GJ, Schneeberger PM, Bevers A, van Ginneken E, Koolen MI (1998) Optimizing screening procedures for Staphylococcus aureus nasal carriage in patients on haemodialysis. Nephrol Dial Transplant 13: 1256-1258. [Crossref]

50. Roubicek C, Brunet P, Mallet MN, Dussol B, Gonzales A, et al. (1995) Nasal carriage of Staphylococcus aureus: prevalence in a hemodialysis center and effect on bacteremia Nephrologie 16: 229-232. [Crossref]

Copyright: (C2015 Fan Z. This is an open-access article distributed under the terms of the Creative Commons Attribution License, which permits unrestricted use, distribution, and reproduction in any medium, provided the original author and source are credited. 